\title{
Recurrence and interoccurrence behavior of self-organized complex phenomena
}

\author{
S. G. Abaimov ${ }^{1}$, D. L. Turcotte ${ }^{1}$, R. Shcherbakov ${ }^{1,2}$, and J. B. Rundle ${ }^{1,2}$ \\ ${ }^{1}$ Department of Geology, University of California, Davis, California, 95616, USA \\ ${ }^{2}$ Center for Computational Science and Engineering, University of California, Davis, California, 95616, USA
}

Received: 5 June 2007 - Revised: 25 July 2007 - Accepted: 25 July 2007 - Published: 2 August 2007

\begin{abstract}
The sandpile, forest-fire and slider-block models are said to exhibit self-organized criticality. Associated natural phenomena include landslides, wildfires, and earthquakes. In all cases the frequency-size distributions are well approximated by power laws (fractals). Another important aspect of both the models and natural phenomena is the statistics of interval times. These statistics are particularly important for earthquakes. For earthquakes it is important to make a distinction between interoccurrence and recurrence times. Interoccurrence times are the interval times between earthquakes on all faults in a region whereas recurrence times are interval times between earthquakes on a single fault or fault segment. In many, but not all cases, interoccurrence time statistics are exponential (Poissonian) and the events occur randomly. However, the distribution of recurrence times are often Weibull to a good approximation. In this paper we study the interval statistics of slip events using a slider-block model. The behavior of this model is sensitive to the stiffness $\alpha$ of the system, $\alpha=k_{C} / k_{L}$ where $k_{C}$ is the spring constant of the connector springs and $k_{L}$ is the spring constant of the loader plate springs. For a soft system (small $\alpha$ ) there are no system-wide events and interoccurrence time statistics of the larger events are Poissonian. For a stiff system (large $\alpha$ ), system-wide events dominate the energy dissipation and the statistics of the recurrence times between these system-wide events satisfy the Weibull distribution to a good approximation. We argue that this applicability of the Weibull distribution is due to the power-law (scale invariant) behavior of the hazard function, i.e. the probability that the next event will occur at a time $t_{0}$ after the last event has a power-law dependence on $t_{0}$. The Weibull distribution is the only distribution that has a scale invariant hazard function. We further show that the onset of system-wide events is a well defined critical point. We find that the number of system-wide events $N_{\mathrm{SWE}}$
\end{abstract}

Correspondence to: S. G. Abaimov

(abaimov@geology.ucdavis.edu) satisfies the scaling relation $N_{\mathrm{SWE}} \propto\left(\alpha-\alpha_{C}\right)^{\delta}$ where $\alpha_{C}$ is the critical value of the stiffness. The system-wide events represent a new phase for the slider-block system.

\section{Introduction}

The discovery (Bak, et al., 1988) that a wide range of models and natural phenomena exhibit "self-organized criticality" has led to many studies (Turcotte, 1999). It should be noted that the relation of critical-point phenomena to these models is controversial (Grassberger, 2002). Type models include the sand pile (Bak, et al., 1988), forest fire (Drossel and Schwabl, 1992), and slider-block models (Carlson and Langer, 1989). Directly related natural phenomena include landslides, wild fires, and earthquakes (Turcotte and Malamud, 2004).

One property of the models and the natural phenomena is that the frequency-magnitude statistics of avalanches are often power-law (fractal) in a robust way (Turcotte, 1999). An explanation for this robust behavior is given in terms of an inverse cascade of metastable clusters (Gabrielov, et al., 1999; Turcotte, et al., 1999; Yakovlev, et al., 2005). A metastable cluster is the region over which an avalanche spreads once triggered. Clusters grow primarily by coalescence. Growth dominates over losses except for the very largest clusters. The cascade of cluster growth is self similar and the frequency-area distributions of both clusters and avalanches are power law.

Another important property of the models and natural phenomena is the interval statistics for the recurrence of events (avalanches) in these models and natural phenomena. In particular, what is the statistical distribution of interval times for large events (peaks over threshold). A number of studies of interval time statistics have been carried out that show the applicability of the exponential (Poissonian) distribution (Sanchez, et al., 2002).

Published by Copernicus Publications on behalf of the European Geosciences Union and the American Geophysical Union. 
A particularly important application of interval time statistics is to earthquakes. In order to study the interval time statistics of earthquakes it is necessary to make a clear distinction between interoccurrence times and recurrence times. Interoccurrence times are the time intervals between earthquakes on all faults in a region. Recurrence times are the time intervals between successive earthquakes on a single fault or fault segment. These are generally referred to as characteristic earthquakes.

We first discuss interoccurrence times. All earthquakes in a specified region and specified time window with magnitudes greater than a specified magnitude are considered to be point events. Based on studies of all earthquakes in southern California during a prescribed time interval, Bak et al. (2002) obtained a universal scaling for the statistical distribution of interoccurrence times. Subsequently, other studies of this type have been carried out (Carbone, et al., 2005; Corral, 2003, 2004a, b, 2005a, b; Davidsen and Goltz, 2004; Lindman, et al., 2005; Livina, et al., 2005a, b). Shcherbakov et al. (2005) showed that this observed behavior for aftershocks can be explained by a non-homogeneous Poisson process. The earthquakes occur randomly but the rate of occurrence is determined by Omori's law for the temporal decay of aftershock activity.

Major faults experience the quasi-periodic occurrence of large earthquakes. These are known as characteristic earthquakes. Available evidence is that there is considerable variability in both the recurrence times and in the magnitudes of characteristic earthquakes. This variability can be attributed to the interactions between faults and fault segments. The statistical distribution of recurrence times is an important input into probabilistic seismic hazard assessments such as the most recent one for the San Francisco Bay region (Working Group on California Earthquake Probabilities, 2003). Several statistical distributions have been proposed for the recurrence times between characteristic earthquakes including the exponential (Poissonian), Weibull, log-normal, and Brownian passage time distributions (Matthews et al., 2002).

In this paper we carry out a study of interval time statistics for a slider-block model. The behavior of this system is controlled by the stiffness parameter $\alpha$ with $\alpha=\frac{k_{C}}{k_{L}}$ where $k_{C}$ is the spring constant of the connector springs and $k_{L}$ is the spring constant of the loader springs. We show that the transition to system-wide slip events occurs at a critical value of $\alpha, \alpha_{C}$. We find that the number of system-wide events $N_{\mathrm{SWE}}$ satisfies the scaling relation $N_{\mathrm{SWE}} \propto\left(\alpha-\alpha_{C}\right)^{\delta}$ for various system sizes. For $\alpha<\alpha_{C}$ there are, on average, no systemwide events and the interval statistics of the larger events are Poisson. We argue that the system-wide events constitute a new phase for the slider-block system. For $\alpha>\alpha_{C}$ we find that the distribution of recurrence time statistics between system-wide events is well approximated by the Weibull distribution.

\section{Weibull distribution}

We will focus our attention on the applicability of the Weibull distribution for the statistical distribution of recurrence times of characteristic events. The cumulative distribution function (cdf) for the Weibull distribution is given by

$P(t)=1-\exp \left[-\left(\frac{t}{\tau}\right)^{\gamma}\right]$

where $P(t)$ is the fraction of the recurrence times that are shorter than $t$, and $\gamma$ and $\tau$ are fitting parameters. The mean $\mu$ and the coefficient of variation $C_{V}$ of the Weibull distribution are given by

$\mu=\tau \Gamma\left(1+\frac{1}{\gamma}\right)$

$C_{V}=\left\{\frac{\Gamma\left(1+\frac{2}{\gamma}\right)}{\left[\Gamma\left(1+\frac{1}{\gamma}\right)\right]^{2}}-1\right\}^{\frac{1}{2}}$

where $\Gamma(x)$ is the gamma function of $x$. If $\gamma=1$ the Weibull distribution becomes the Poisson distribution and with $\gamma=2$ it is the Rayleigh distribution. In the range $0<\gamma<1$ the Weibull distribution is often referred to as the stretched exponential distribution.

An important property of the Weibull distribution is the power-law behavior of the hazard function

$h\left(t_{0}\right)=\frac{\mathrm{pdf}}{1-\mathrm{cdf}}=\frac{\gamma}{\tau}\left(\frac{t_{0}}{\tau}\right)^{\gamma-1}$

The hazard function $h\left(t_{0}\right)$ is the pdf that an event will occur at a time $t_{0}$ after the occurrence of the last event. For the Poisson distribution, $\gamma=1$, the hazard function is constant $h\left(t_{0}\right)=\tau^{-1}$ as expected. For $\gamma>1$ the hazard rate increases as a power of the time $t_{0}$.

For characteristic earthquakes it is expected that the hazard function must increase as the time since the last characteristic earthquake $t_{0}$ increases (Davis, et al., 1989; Sornette and Knopoff, 1997). For this to be the case the tail of the distribution must be thinner than the exponential distribution. For the log-normal distribution the tail is thicker than the exponential and the hazard function decreases with time $t_{0}$. For the Brownian passage time distribution the tail is exponential for large times and the hazard function becomes constant. The Weibull distribution with $\gamma>1$ is the only distribution that has been applied to characteristic earthquakes with an increasing hazard function with increasing $t_{0}$.

Many authors have applied Weibull statistics to distributions of recurrence times between characteristic earthquakes (Hagiwara, 1974; Rikitake, 1976, 1982, 1991; Utsu, 1984). Probably the best studied sequence of characteristic earthquakes is the sequence that occurred on the Parkfield, California section of the San Andreas fault between 1857 and 
2004 (Bakun, et al., 2005). This is because the slip rate is relatively high $(\approx 30 \mathrm{~mm} /$ year $)$ and the earthquakes are relatively small $(m \approx 6.0)$, thus the recurrence times are relatively short ( $\approx 25$ years). Slip on the Parkfield section of the San Andreas fault occurred during $m \approx 6$ earthquakes that occurred in 1857, 1881, 1901, 1922, 1934, 1966, and 2004. The mean and coefficient of variation of these recurrence times are $\mu=24.5$ years, and $C_{V}=0.378$, respectively. Taking these values, the corresponding fitting parameters for the Weibull distribution are $\tau=27.4$ years and $\gamma=2.88$. A second set of characteristic earthquakes on the San Andreas fault have been obtained from paleoseismicity studies at Pallett Creek, California by Sieh et al. 1989. These studies indicated that the intervals between great Southern California earthquakes on the San Andreas fault have approximately the values 44, 63, 67, 134, 200, 246, and 332 years. These authors fit a Weibull distribution to this data and found that $\tau=166.1 \pm 44.5$ years and $\gamma=1.50 \pm 0.80$. Although the fits of the Weibull distribution were quite good in both these cases, the number of events were not sufficient to establish the validity of the Weibull distribution over alternative distributions (Savage, 1994).

In order to provide a larger data base, several numerical simulations of earthquake statistics have been carried out (Goes and Ward, 1994; Rundle, 1988; Rundle, et al., 2004; Ward, 1996, 2000). We give results for the Virtual California simulation (Yakovlev, et al., 2006). This model is a geometrically realistic numerical simulation of earthquakes occurring on the San Andreas fault system. It includes the major strikeslip faults in California and is composed of 650 fault segments, each with a width of $10 \mathrm{~km}$ and a depth of $15 \mathrm{~km}$. The fault segments interact with each other elastically utilizing dislocation theory. Virtual California is a backslip model, the accumulation of a slip deficit on each segment is prescribed using available data. The mean recurrence times of earthquakes on each segment are also prescribed using available data to give friction law parameters. The statistical distribution of recurrence times on the northern San Andreas fault (site of the 1906 San Francisco earthquake) was obtained from a 1000000 year simulation. The mean recurrence time for 4606 simulated earthquakes with $M>7.5$ on this section is $\mu=217$ years and the coefficient of variation is $C_{V}=0.528$. The corresponding fitting parameters for the Weibull distribution are $\tau=245$ years and $\gamma=1.97$. Yakovlev et al. (2006) showed that the Weibull distribution fit the data significantly better than the alternative log-normal and Brownian passage time distributions. Taking the above values the hazard function from Eq. (4) for the next great San Francisco earthquake $\left(t_{0}=100\right.$ years $)$ is $h(100$ years $)=3.3 \times 10^{-3}$ year $^{-1}$. This is the estimated probability that an earthquake with a magnitude greater than 7.5 will occur on the San Andreas fault near San Francisco in the next year.

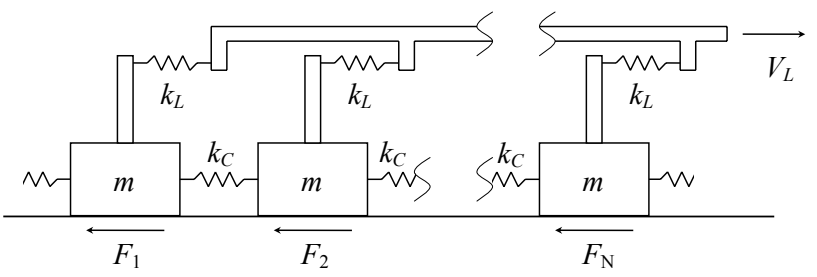

Fig. 1. Illustration of our one-dimensional slider-block model. A linear array of $N$ blocks of mass $m$ are pulled along a surface by a constant velocity $V_{L}$ loader plate. The loader plate is connected to each block with a loader spring with spring constant $k_{L}$ and adjacent blocks are connected by springs with spring constant $k_{C}$. The frictional resisting forces are $F_{1}, F_{2}, \ldots, F_{N}$.

\section{Slider-block model}

In this paper we consider the behavior of a slider-block model in order to study the statistics of the interval times between slip events. We utilize a variation of the linear sliderblock model which Carlson and Langer (1989) used to illustrate the self-organization of such models. We consider a linear chain of 25, 50, and 100 slider blocks of mass $m$ pulled over a surface at a constant velocity $V_{L}$ by a loader plate as illustrated in Fig. 1. Each block is connected to the loader plate by a spring with spring constant $k_{L}$. Adjacent blocks are connected to each other by springs with spring constant $k_{C}$. Boundary conditions are assumed to be periodic: the last block is connected to the first one.

The blocks interact with the surface through friction. In this paper we prescribe a static-dynamic friction law. The static stability of each slider-block is given by

$k_{L} y_{i}+k_{C}\left(2 y_{i}-y_{i-1}-y_{i+1}\right)<F_{S i}$

where $F_{S i}$ is the maximum static friction force on block $i$ holding it motionless, and $y_{i}$ is the position of block $i$ relative to the loader plate.

During strain accumulation due to loader plate motion all blocks are motionless relative to the surface and have the same increase of their coordinates relative to the loader plate

$\frac{d y_{i}}{d t}=V_{L}$

When the cumulative force of the springs connecting to block $i$ exceeds the maximum static friction $F_{S i}$, the block begins to slide. We include inertia, and the dynamic slip of block $i$ is controlled by the equation

$m \frac{d^{2} y_{i}}{d t^{2}}+k_{L} y_{i}+k_{C}\left(2 y_{i}-y_{i-1}-y_{i+1}\right)=F_{D i}$

where $F_{D i}$ is the dynamic (sliding) frictional force on block $i$. The loader plate velocity is assumed to be much smaller than the slip velocity, requiring

$V_{L} \ll \frac{F_{S}^{r e f}}{\sqrt{k_{L} m}}$ 


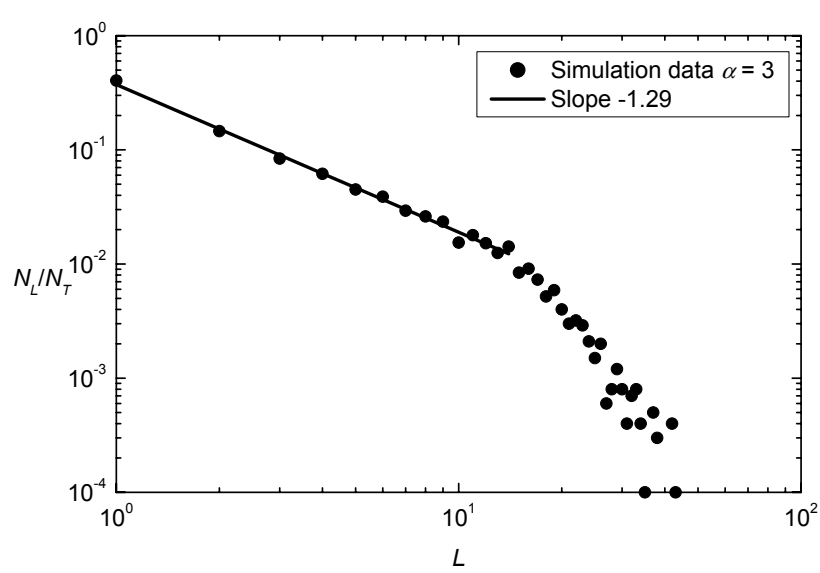

Fig. 2. Frequency-size distribution of 10000 slip events for a "soft" system with $\alpha=3$. The ratio of the number of events $N_{L}$ of linear size $L$ to the total number of events $N_{T}$ is given as a function of $L$. The solid line is a power-law dependence with exponent -1.29 .

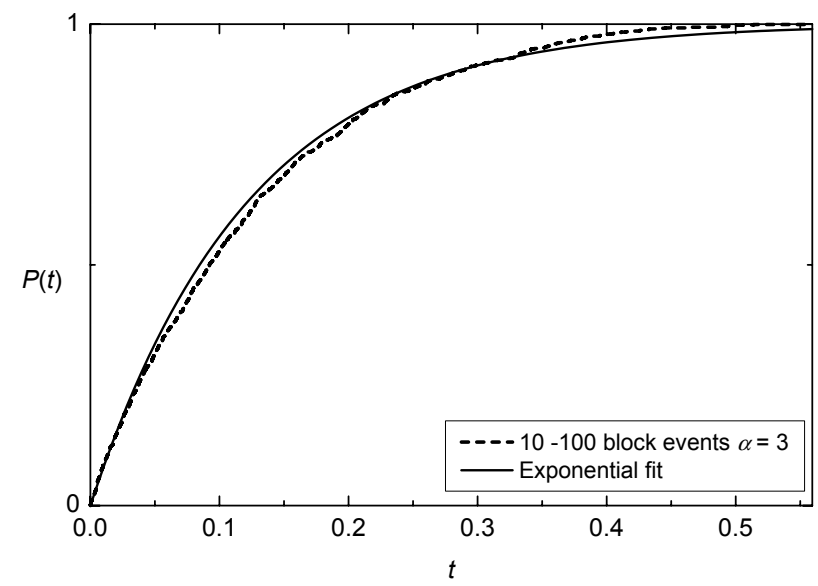

Fig. 3. Cumulative distribution function $P(t)$ of interoccurrence times $t$ for the events given in Fig. 2 in the size range $L=10$ to 100 . The dashed line is the distribution of observed recurrence times. The continuous line is the best-fit exponential (Poisson) distribution.

so the movement of the loader plate is neglected during a slip event. The sliding of one block can trigger the instability of the other blocks forming a many block event. When the velocity of a block is zero it sticks with zero velocity if the static friction criterion (5) is satisfied, if the criterion is not satisfied the block continues to slip.

It is convenient to introduce the nondimensional variables and parameters

$$
\tau_{f}=t \sqrt{\frac{k_{L}}{m}}, \tau_{s}=\frac{t k_{L} V_{L}}{F_{S}^{\text {ref }}}, Y_{i}=\frac{k_{L} y_{i}}{F_{S}^{\text {ref }}}, \phi=\frac{F_{S i}}{F_{D i}}, \alpha=\frac{k_{C}}{k_{L}}, \beta_{i}=\frac{F_{S i}}{F_{S}^{\text {ref }}}
$$

The ratio of static to dynamic friction $\phi$ is assumed to be the same for all blocks but the values themselves $\beta_{i}$ vary from block to block with $F_{S}^{\text {ref }}$ as a reference value of the static frictional force ( $F_{S}^{\text {ref }}$ is the minimum value of all $F_{S i}$ ). Stress accumulation occurs during the slow time $\tau_{s}$ when all blocks are stable, and slip of blocks occurs during the fast time $\tau_{f}$ when the loader plate is assumed to be approximately motionless.

In terms of these nondimensional variables the static stability condition (5) becomes

$Y_{i}+\alpha\left(2 Y_{i}-Y_{i-1}-Y_{i+1}\right)<\beta_{i}$

the strain accumulation Eq. (6) becomes

$\frac{d Y_{i}}{d \tau_{S}}=1$

and the dynamic slip Eq. (7) becomes

$\frac{d^{2} Y_{i}}{d \tau_{f}^{2}}+Y_{i}+\alpha\left(2 Y_{i}-Y_{i-1}-Y_{i+1}\right)=\frac{\beta_{i}}{\phi}$

Before obtaining solutions, it is necessary to prescribe the parameters $\phi, \alpha$, and $\beta_{i}$. The parameter $\alpha$ is the stiffness of the system. We first consider the 100 block system and obtain solutions for $\alpha$ from 3 to 1000 in this paper. For $\alpha=3$ the system is soft and there are no system wide (100 block) events. For $\alpha=1000$ the system is stiff and system wide (100 block) events dominate. The ratio $\phi$ of static friction to dynamic friction is taken to be the same for all blocks $\phi=1.5$, while the values of frictional parameters $\beta_{i}$ are assigned to blocks by uniform random distribution from the range $1<\beta_{i}<3.5$. This random variability in the system is a "noise" required to generate event variability in stiff systems.

The loader plate springs of all blocks extend according to Eq. (11) until a block becomes unstable from Eq. (10). The dynamic slip of that block is calculated using the RungeKutta numerical method to obtain a solution of Eq. (12). A coupled 4th-order iterational scheme is used, and all equations are solved simultaneously (the Runge-Kutta coefficients of neighboring blocks participate in the generation of the next order Runge-Kutta coefficient for the given block). The dynamic slip of one block may trigger the slip of other blocks and the slip of all blocks is followed until they all become stable. Then the procedure repeats.

We first give results for a soft system with $\alpha=3$. The linear event size $L$ is the number of blocks that participate in the event. Frequency-size statistics for 10000 events are given in Fig. 2. The smaller events in the range 1-14 satisfy a powerlaw relation with exponent -1.29 to a reasonable approximation. For event sizes greater than $L=14$ there is a roll over and there are no system-wide (100 block) events. We next consider the interoccurrence time statistics for events. In order to do this we specify a threshold magnitude and consider events only larger than this threshold. The cumulative distribution $P(t)$ of interval times for events in the size range from $L=10$ to 100 is given in Fig. 3. Also included in this figure is the exponential (Poisson) fit to these data, this is Eq. (1) with $\gamma=1$. To a good approximation the events occur randomly 


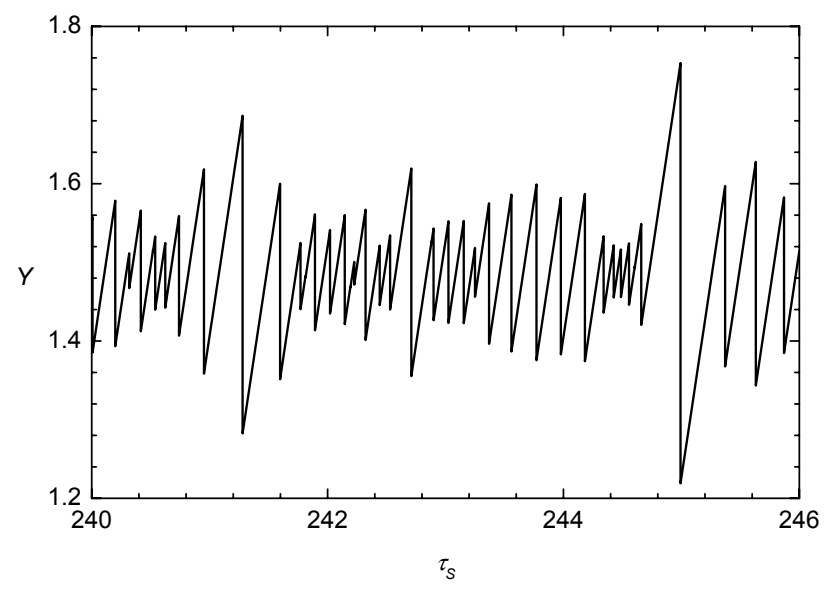

Fig. 4. The nondimensional position of a typical block $Y$ is given as a function of the nondimensional slow time $\tau_{S}$. This is a result for a heterogeneous stiff system with $\alpha=1000$.

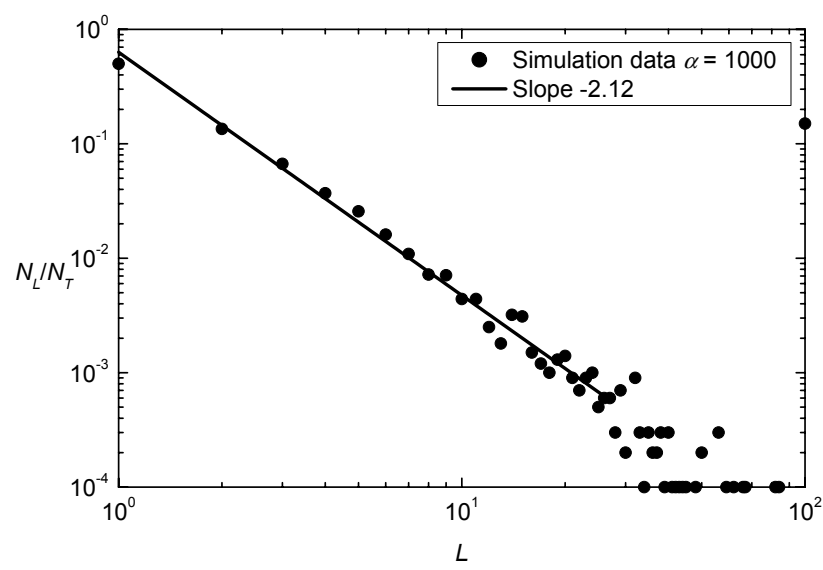

Fig. 5. Frequency-size distribution of 10000 slip events for a "stiff" system with $\alpha=1000$. The ratio of the number of events $N_{L}$ of linear size $L$ to the total number of events $N_{T}$ is given as a function of $L$. The solid line is a power-law dependence with exponent -2.12 .

and are not correlated. A similar result was obtained for the sandpile model (Sanchez, et al., 2002). Since these smaller events occur at different positions along the array they do not have a memory of previous events.

We next give results for a stiff system with $\alpha=1000$. With infinite stiffness the system will behave as a rigid body with periodic slip events. The stiff homogeneous system with $\beta_{i}=1$ shows a "creeping" behavior in our simulations - it creeps block by block - only a small number of blocks move simultaneously at any time. The introduction of even a small amount of symmetry-breaking heterogeneity causes systemwide (100 block) events to occur.

For the heterogeneous stiff system with a random distribution of friction coefficients $1<\beta_{i}<3.5$ the motion organizes itself into the recurrence of system-wide (100 block)

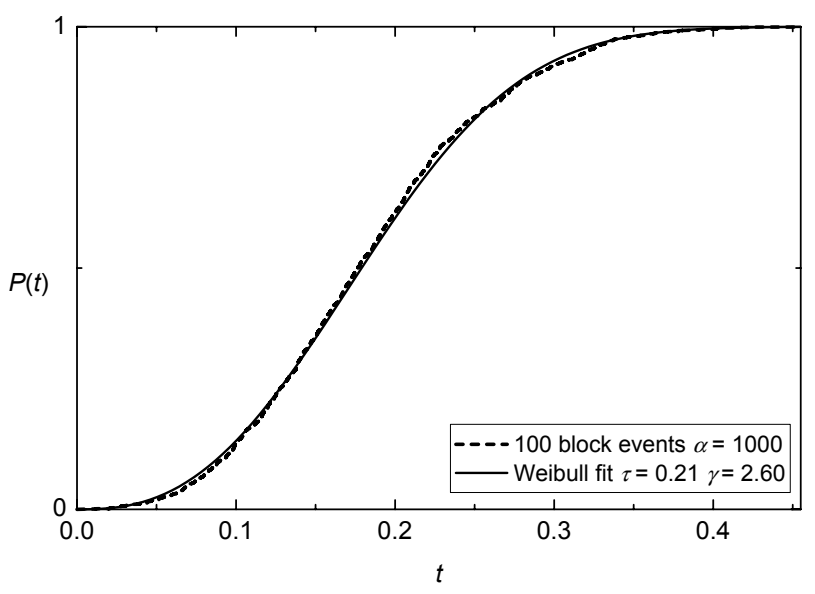

Fig. 6. Cumulative distribution function $P(t)$ of recurrence times $t$ for the 1500 system-wide (100 block) events with $\alpha=1000$. The dashed line is the distribution of observed recurrence times. The continuous line is the best-fit Weibull distribution with $\tau=0.21$ and $\gamma=2.60$.

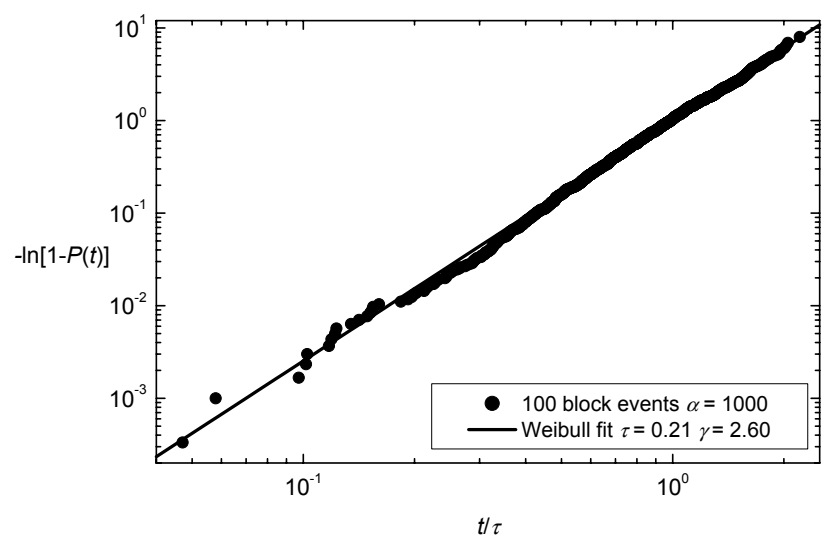

Fig. 7. Weibull probability plot of the cumulative distribution of recurrence times for the data given in Fig. 6. The solid line corresponds to a Weibull distribution as given in Eq. (13) with $\tau=0.21$ and $\gamma=2.60$.

events separated by sets of small size events. A typical example is given in Fig. 4. Frequency-size statistics for 10000 events are given in Fig. 5. Again the smaller events in the range 1-27 satisfy a power-law relation with exponent -2.12 to a reasonable approximation. In this case there are 1500 system-wide (100 block) events. We consider that these are equivalent to characteristic earthquakes. We next consider the recurrence time statistics for these events. The cumulative distribution of recurrence times is given in Fig. 6. Also included in this figure is the fit of the Weibull distribution (1) to this data obtained by taking $\tau=0.21$ and $\gamma=2.60$. It is standard practice to test the validity of a Weibull distribution 


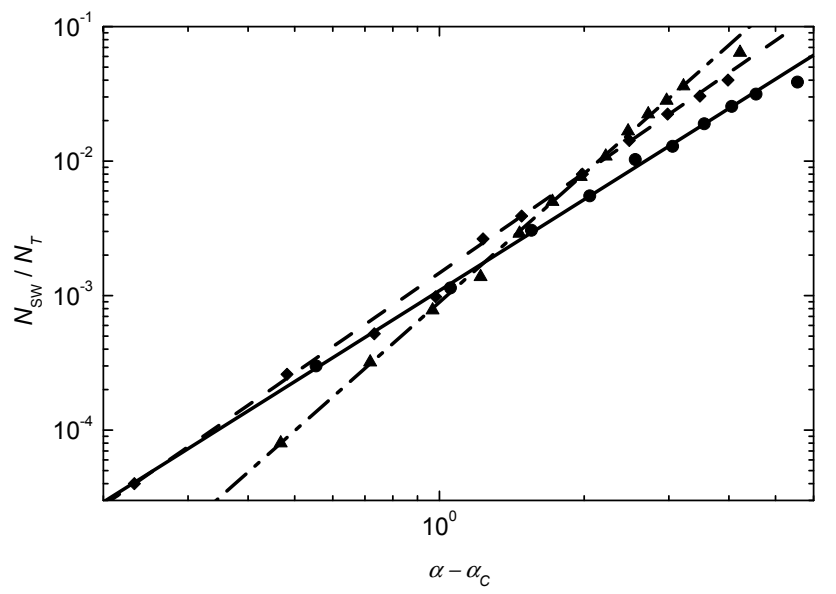

Fig. 8. Dependence of simulation values of the fraction of events that are system-wide $N_{S W} / N_{T}$ on the difference between the stiffness of the array $\alpha$ and the critical value of this tuning parameter $\alpha_{C}$. The circles are the results for a 100 block simulation and the solid line is the power-law scaling from Eq. (14) taking $\alpha_{C}=4.45$ and $\delta=2.25$. The diamonds are the results for a 50 block simulation and the dashed line is the power-law scaling from Eq. (14) taking $\alpha_{C}=2.518$ and $\delta=2.47$. The triangles are the results for a 25 block simulation and the dashed-dot line is the power-law scaling from Eq. (14) taking $\alpha_{C}=0.782$ and $\delta=3.18$.

using a "Weibull probability plot". We rewrite Eq. (1) as

$$
-\ln (1-P(t))=\left(\frac{t}{\tau}\right)^{\gamma}
$$

In Fig. 7 we plot $\log [-\ln (1-P(t))]$ versus $\log \left[\frac{t}{\tau}\right]$ for our data. The Weibull distribution requires a straight-line fit with slope $\gamma$. Taking $\tau=0.21$ and $\gamma=2.60$ the fit shown has $R^{2}=0.998$. The data are well approximated by the straightline fit.

We have obtained results for other values of the stiffness $\alpha$. We find that the number of system wide events is virtually independent of $\alpha$ in the range $50<\alpha<10000$. We also find that the recurrence time statistics satisfy the Weibull distribution to a good approximation in this range. The Weibull exponent $\gamma$ slowly decreases from $\gamma=3.3$ at $\alpha=100$ to $\gamma=2.2$ at $\alpha=10000$. We have also examined the hypothesis that the onset of system-wide events is a critical point (Grassberger, 2002) and that $\alpha$ is the relevant tuning parameter. We first consider the ratio of the number of system-wide slip events $N_{S W}$ to the total number of all slip events $N_{T}$. We test the power-law relation

$$
\frac{N_{S W}}{N_{T}} \propto\left(\alpha-\alpha_{C}\right)^{\delta}
$$

with $\alpha>\alpha_{C}$.

In order to test the validity of this relation we give results for linear chains of 25, 50, and 100 slider blocks. In Fig. 8 we give the dependence $N_{S W} / N_{T}$ versus $\alpha-\alpha_{C}$ for

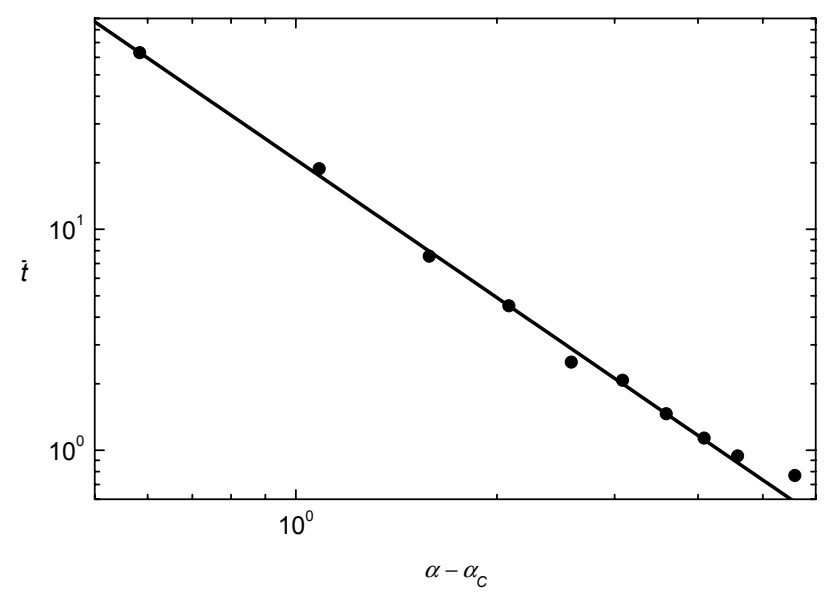

Fig. 9. Dependence of simulation values of the mean nondimensional time between system-wide events $\bar{t}$ on the difference between the stiffness of the array $\alpha$ and the critical value of this tuning parameter $\alpha_{C}=4.42$ (solid circles). The straight line is the power-law scaling from Eq. (15) taking the exponent $\varepsilon=2.07$.

the three cases. For 25 slider blocks the simulation results are well approximated $\left(R^{2}=0.999\right)$ by this scaling relation taking $\alpha_{C}=0.782 \pm 0.164$ and $\delta=3.18 \pm 0.28$ (the errors are $95 \%$ confidence bounds). For 50 slider blocks the simulation results are well approximated $\left(R^{2}=0.998\right)$ by Eq. (14) taking $\alpha_{C}=2.518 \pm 0.182$ and $\delta=2.47 \pm 0.28$. And for 100 slider blocks the simulation results are again well approximated $\left(R^{2}=0.998\right)$ by Eq. (14) taking $\alpha_{C}=4.45 \pm 0.46$ and $\delta=2.25 \pm 0.52$. The good agreement of our simulation results with the power-law scaling relation (14) is evidence that the onset of system-wide events is a critical point with the stiffness $\alpha$ as the tuning parameter. The system-wide events represent a new phase and the critical point is the transition to this phase.

To further test the power-law scaling in the vicinity of a critical point we test two other scaling laws for the 100 sliderblock model. We next consider the mean recurrence time between system-wide events $\bar{t}$. We test the scaling relation

$\bar{t} \propto\left(\alpha-\alpha_{C}\right)^{-\varepsilon}$

with $\alpha>\alpha_{C}$. In Fig. 9 we show that our simulations are well approximated $\left(R^{2}=0.999\right)$ by this scaling relation again taking $\alpha_{C}=4.42 \pm 0.14$ and in this case $\varepsilon=2.07 \pm 0.29$. We finally consider the ratio of the energy dissipated in system-wide events to the energy dissipated in all events $e_{100} / e_{T}$. We test the scaling relation

$\frac{e_{100}}{e_{T}} \propto\left(\alpha-\alpha_{C}\right)^{\mu}$

with $\alpha>\alpha_{C}$. In Fig. 10 we show that our simulations are well approximated $\left(R^{2}=0.998\right)$ by this scaling relation taking $\alpha_{C}=4.57 \pm 0.28$ and in this case $\mu=1.78 \pm 0.30$. The validity of these power-law scaling relations indicates that the onset 
of system-wide events is a critical point (Goldenfeld, 1992; Stauffer and Aharony, 1992).

The three values of the critical stiffness $\left(\alpha_{C}=4.45 \pm 0.46\right.$, $\alpha_{C}=4.42 \pm 0.14$, and $\alpha_{C}=4.57 \pm 0.28$ ) should be equal. The differences are much smaller than the $95 \%$ confidence limits. Taking the mean value we obtain $\alpha_{C}=4.48 \pm 0.17$. Another question is whether the three exponents $(\delta=2.25 \pm 0.52$, $\varepsilon=2.07 \pm 0.29$, and $\mu=1.78 \pm 0.30$ ) should be equal. Again the differences are less than the $95 \%$ confidence limits. The product of the number of system-wide slip events $N_{100}$ and the mean recurrence time between system-wide events $\bar{t}$ equals the total time of the simulation - a quantity which does not have a singularity and is a constant in the vicinity of the critical point. This suggests that the exponents $\delta$ and $\varepsilon$ should be equal. In the same way the ratio of the energy dissipated in system-wide events during the simulation $e_{100}$ to the number of system-wide slip events $N_{100}$ is the mean energy dissipated in one system-wide event - also a quantity which does not have a singularity and is a constant in the vicinity of the critical point from above. This suggests that the exponents $\delta$ and $\mu$ should also be equal. Assuming that the three exponents are equal we find $\delta=\varepsilon=\mu=2.03 \pm 0.21$.

One way to interpret the results we have obtained is in terms of finite-size scaling (Goldenfeld, 1992; Stauffer and Aharony, 1992). Specifically, we consider finite-size scaling within the context of clusters observed in percolation theory. Here the system size has length $L$, and the clustering of events is associated with the appearance of a correlation length $\xi$. The system boundaries have little or no influence on the problem when $\xi \ll L$. However, when $\xi>L$, system boundaries introduce cutoffs in cluster size, and are therefore expected to influence the values of percolation thresholds, scaling exponents, and other observed quantities. For example, the specific values $\alpha_{C}$ are affected by the size $L=25,50$, and 100 that is used. When $\xi \sim L$, a variety of crossover effects may be observed.

The behavior of the system depends upon the ratio of the system size to the correlation length. Finite-size effects play a crucial role here, that's why we investigate the model with only 100 slider-blocks. Changing the stiffness of the system as a tuning parameter is equivalent to changing the ratio of the system size to the correlation length. The critical point is the result of the appearance of system-wide events, and a consequence is the change of the interval statistics from the Poissonian (exponential) below the critical point to the Weibull statistics in the limit of very stiff system.

\section{Why Weibull}

A primary focus of this paper is on the applicability of the Weibull distribution to the statistics of recurrence times for self-organizing complex phenomena. In the engineering literature this distribution has found wide applicability to the statistics of failure. A standard failure problem is that of

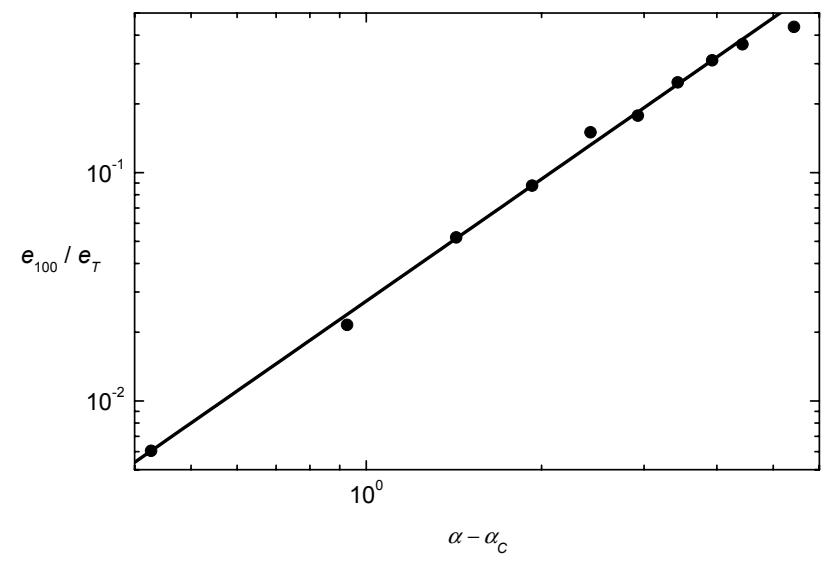

Fig. 10. Dependence of simulation values of the fraction of the energy dissipated in system-wide events $e_{100} / e_{T}$ on the difference between the stiffness of the array $\alpha$ and the critical value of this tuning parameter $\alpha_{C}=4.57$ (solid circles). The straight line is the power-law scaling from Eq. (16) taking the exponent $\mu=1.78$.

fiber bundles which can be associated with composite materials (Newman and Phoenix, 2001). The dynamic failure of a bundle of $N_{0}$ fibers is modeled empirically by the relation

$\frac{d N}{d t}=-N v_{r}\left(\frac{\sigma}{\sigma_{r}}\right)^{p}$

where $N$ is the number of remaining fibers and $v_{r}$ is the reference hazard rate at the reference stress $\sigma_{r}$. Assuming that the stress increases linearly in time

$$
\frac{\sigma}{\sigma_{r}}=\frac{t}{t_{r}}
$$

Eq. (17) becomes

$\frac{d N}{d t}=-N v_{r}\left(\frac{t}{t_{r}}\right)^{p}$

Integrating with $N=N_{0}$ at $t=0$ gives

$$
\frac{N}{N_{0}}=\exp \left[-\frac{v_{r} t^{p+1}}{p \tau^{p}}\right]
$$

We next introduce

$$
P(t)=1-\frac{N}{N_{0}}, \gamma=p+1, v_{r}=\frac{\gamma}{\tau}
$$

where $P(t)$ is the cumulative distribution function of failure. Substituting Eq. (21) into Eq. (20) gives the Weibull distribution Eq. (1). The self-similar (power-law) dependence of the hazard rate on time leads directly to the Weibull distribution of failure times.

The hazard function as given in Eq. (4) for the Weibull distribution has a power-law dependence on the time since the last event. The stress on a fault is expected to increase linearly in time due to the steady motion of the tectonic plates 
just as the forces on our slider blocks increase linearly in time due to the steady motion of the loader plate. Similarly the stress on the fiber bundle as given by Eq. (18) increases linearly with time. We have shown above that the failure rate of a fiber bundle modeled by Eq. (17) gives a Weibull distribution. This provides a basis for the application of the Weibull distribution both to our slider-block model and to characteristic earthquakes.

\section{Fractional noises}

Another application of the Weibull (stretched exponential) distribution is to fractional noises. Fractional noises and walks are time series with long range power-law correlations. This dependence is generally quantified in terms of a powerlaw dependence of the power-spectral density $S$ on frequency $f$ (Malamud and Turcotte, 1999)

$S \propto f^{-\beta}$

For a stationary fractional noise we have $-1 \leq \beta \leq 1$. In the range $0<\beta \leq 1$ adjacent values are correlated, large (small) follows large (small). In the range $-1 \leq \beta<0$ adjacent values are anticorrelated, small (large) follows large (small). Quantification of correlations in a fractional noise is done in terms of the autocorrelation function $r(s)$ (Altmann, et al., 2004; Altmann and Kantz, 2005; Bunde, et al., 2003, 2004; Pennetta, 2006)

$r(s) \propto s^{-\gamma}$

where $s$ is the lag. The exponents $\gamma$ and $\beta$ are related by

$\gamma=1-\beta$

For a stationary fractional noise we have $0<\gamma<2$. In the range $0<\gamma<1$ adjacent values are correlated and in the range $1<\gamma<2$ adjacent values are anticorrelated.

Another alternative quantification of the scaling properties of a fractional noise is in terms of rescaled range (R/S) analysis. In this case we have (Malamud and Turcotte, 1999)

$\frac{R}{S} \propto \tau^{H u}$

where $\tau$ is the length of the record and $H u$ is the Hurst exponent. The three power-law exponents $H u, \beta$, and $\gamma$ are related by

$H u=\frac{\beta+1}{2}=\frac{2-\gamma}{2}$

For a stationary fractional noise we have $0<H u<1$. In the range $0<H u<0.5$ adjacent values are anticorrelated and in the range $0.5<H u<1$ adjacent values are correlated.

For an uncorrelated white-noise time series we have $\beta=0$, $H u=0.5$, and $\gamma=1$. For many natural time series it is found that $0.70 \leq H u \leq 0.80$ (Pelletier and Turcotte, 1999). Examples include river discharges, lake levels, varve thicknesses, sunspot numbers, and atmospheric time series. The corresponding values of the exponents $\beta$ and $\gamma$ are: $0.40 \leq \beta \leq 0.60$ and $0.40 \leq \gamma \leq 0.60$.

Recent studies have been carried out on the recurrence statistics of peaks over thresholds for fractional noises (Altmann, et al., 2004; Altmann and Kantz, 2005; Bunde, et al., 2003, 2004; Pennetta, 2006). These studies have shown that the recurrence statistics satisfy the Weibull (stretched exponential) distribution given in Eq. (1). The exponent $\gamma$ in the Weibull distribution is equal to exponent for the autocorrelation function given in Eq. (23).

For an uncorrelated white noise with $\gamma=1$ we have a Poisson (exponential) distribution from Eq. (1) as expected and the hazard rate from Eq. (4) is constant. For a correlated noise we have $0<\gamma<1$ and the Weibull distribution (1) is a stretched exponential with a hazard function from Eq. (4) that has a power-law decrease with time. For an anticorrelated noise we have $1<\gamma<2$ and the hazard function from Eq. (4) has a power-law increase with time.

\section{Conclusions}

One purpose of this paper is to demonstrate that the interval statistics of both models and natural systems exhibit Weibull distributions. We argue that the reason for the applicability of the Weibull distribution is that its hazard function as defined in Eq. (4) is a scale-invariant power-law function of time. It is important to make a clear distinction between the pdf of interval times for all events and the hazard function which is the pdf of the interval time for a single event. There is a mean interval for all events and for the Weibull distribution this has been given in Eq. (2). The distribution of all interval times is clearly scale dependent. Instead, consider the behavior of the system after an event has occurred. The pdf of possible interval times until the next event is the hazard function. The Weibull distribution is the only distribution that gives a power-law, scale-invariant distribution of these intervals.

In order to demonstrate the applicability of the Weibull distribution we have considered the behavior of a sliderblock model. We have carried out a full dynamic simulation using linear arrays of slider blocks. The behavior of the model is governed by its "stiffness" $\alpha$, the ratio of the connector spring constant to the loader spring constant as given in Eq. (9). For low values of $\alpha$, soft systems, there are no system-wide events. For high values of $\alpha$, stiff systems, large number of system-wide events occur in which all blocks slip simultaneously. In order to get system-wide events it was necessary to break the symmetry of the model by introducing a random variability of the friction coefficients.

We find that the statistical distribution of recurrence times between system-wide events of the 100 block system with $\alpha=1000$ satisfies a Weibull distribution to a very good approximation. We have also considered the distribution of interoccurrence times of large events for a soft system with 
$\alpha=3$. In this case the distribution is well approximated by an exponential (Poisson) distribution. An event does not have a significant "memory" of prior events. This is consistent with prior studies of SOC models such as the sandpile model (Sanchez, et al., 2002) in which exponential distributions were found.

There have been many papers published on a variety of slider-block models (Turcotte, 1999). In order to reduce computer time almost all utilize a cellular automata approximation, only one block is allowed to slip instead of multipleblock slip events. We have chosen to use a fully dynamic simulation with a relatively small number (100) of blocks. We believe our results are robust both in terms of Weibull statistics and in terms of a well defined critical point.

We argue that there is a direct analogy between the behavior of our slider-block model and the behavior of actual earthquakes. Characteristic earthquakes are earthquakes that repeatedly occur on a particular fault or fault segment. There is considerable evidence from both actual earthquakes (Hagiwara, 1974; Rikitake, 1976, 1982, 1991; Utsu, 1984) and simulations (Yakovlev, et al., 2006) that the distribution of recurrence times for a set of characteristic earthquakes on a fault satisfies a Weibull distribution. This is in direct analogy to the behavior of our stiff slider-block model. There is also observational evidence that the interoccurrence times between all earthquakes in a region (on many different faults) satisfy Poissonian statistics (Shcherbakov, et al., 2005). This is in direct analogy to the behavior of our soft slider-block model.

Weibull (stretched exponential) statistics have also been found to be applicable to the distribution of peaks over threshold for fractional Gaussian noises (Altmann, et al., 2004; Altmann and Kantz, 2005; Bunde, et al., 2003; 2004; Pennetta, 2006). Again we argue that this is due to the scaleinvariant (power-law) dependence of the hazard function for the Weibull distribution. For correlated fractional noises the probability of the next event decreases as an inverse power of the time $t_{0}$ since the last event as given in Eq. (4). For anticorrelated fractional noises the probability of the next event increases as a power of the time since the last event. This is the behavior associated with characteristic earthquakes and with our "stiff" slider-block simulations.

Acknowledgements. We wish to acknowledge valuable discussions with G. Yakovlev and B. Newman. This work has been supported by NSF Grants ATM 0327571 and ATM 0327799.

Edited by: P. Yiou

Reviewed by: two anonymous referees

\section{References}

Altmann, E. G., da Silva, E. C., and Caldas, I. L.: Recurrence time statistics for finite size intervals, Chaos, 14, 975-981, 2004.
Altmann, E. G. and Kantz, H.: Recurrence time analysis, longterm correlations, and extreme events, Phys. Rev. E, 71, 056106, doi:10.1103/PhysRevE.71.056106, 2005.

Bak, P., Christensen, K., Danon, L., and Scanlon, T.: Unified scaling law for earthquakes, Phys. Rev. Lett., 88, 178501, doi: 10.1103/PhysRevLett.88.178501, 2002.

Bak, P., Tang, C., and Wiesenfeld, K.: Self-organized criticality, Phys. Rev. A, 38, 364-374, 1988.

Bakun, W. H., Aagard, B., Dost, B., et al.: Implications for prediction and hazard assessment from the 2004 Parkfield earthquake, Nature, 437, 969-974, 2005.

Bunde, A., Eichner, J. F., Havlin, S., and Kantelhardt, J. W.: The effect of long-term correlations on the return periods of rare events, Physica A, 330, 1-7, 2003.

Bunde, A., Eichner, J. F., Havlin, S., and Kantelhardt, J. W.: Return intervals of rare events in records with long-term persistence, Physica A, 342, 308-314, 2004.

Carbone, V., Sorriso-Valvo, L., Harabaglia, P., and Guerra, I.: Unified scaling law for waiting times between seismic events, Europhys. Lett., 71, 1036-1042, 2005.

Carlson, J. M. and Langer, J. S.: Mechanical model of an earthquake fault, Phys. Rev. A, 40, 6470-6484, 1989.

Corral, A.: Local distributions and rate fluctuations in a unified scaling law for earthquakes, Phys. Rev. E, 68, 035102(R), doi:10.1103/PhysRevE.68.035102, 2003.

Corral, A.: Long-term clustering, scaling, and universality in the temporal occurrence of earthquakes, Phys. Rev. Lett., 92, 108501, doi:10.1103/PhysRevLett.92.108501, 2004a.

Corral, A.: Universal local versus unified global scaling laws in the statistics of seismicity, Physica A, 340, 590-597, 2004b.

Corral, A.: Mixing of rescaled data and Bayesian inference for earthquake recurrence times, Nonlin. Processes Geophys., 12, 89-100, 2005a.

Corral, A.: Time-decreasing hazard and increasing time until the next earthquake, Phys. Rev. E, 71, 017101, doi:10.1103/PhysRevE.71.017101, 2005b.

Davidsen, J. and Goltz, C.: Are seismic waiting time distributions universal?, Geophys. Res. Lett., 31, L21612, doi:10.1029/2004GL020892, 2004.

Davis, P. M., Jackson, D. D., and Kagan, Y. Y.: The longer it has been since the last earthquake, the longer the expected time till the next, Bull. Seism. Soc. Am., 79, 1439-1456, 1989.

Drossel, B. and Schwabl, F.: Self-organized critical forest-fire model, Phys. Rev. Lett., 69, 1629-1632, 1992.

Gabrielov, A., Newman, W. I., and Turcotte, D. L.: Exactly soluble hierarchical clustering model: Inverse cascades, self-similarity, and scaling, Phys. Rev. E, 60, 5293-5300, 1999.

Goes, S. D. B. and Ward, S. N.: Synthetic seismicity for the San Andreas Fault, Ann. Geofisica, 37, 1495-1513, 1994.

Goldenfeld, N.: Lectures on Phase Transitions and the Renormalization Group, Addison Wesley, Reading, MA, 394pp., 1992.

Grassberger, P.: Critical behaviour of the Drossel-Schwabl forest fire model, New J. Phys., 4, 17, doi:10.1088/1367-2630/4/1/317, 2002.

Hagiwara, Y.: Probability of earthquake occurrence as obtained from a Weibull distribution analysis of crustal strain, Tectonophys., 23, 313-318, 1974.

Lindman, M., Jonsdottir, K., Roberts, R., Lund, B., and Bodvarsson, R.: Earthquakes descaled: On waiting time dis- 
tributions and scaling laws, Phys. Rev. Lett., 94, 108501, doi:10.1103/PhysRevLett.94108501, 2005.

Livina, V., Havlin, S., and Bunde, A.: Memory in the occurrence of earthquakes, Phys. Rev. Lett., 95, 208501, doi:10.1103/PhysRevLett.95.208501, 2005a.

Livina, V., Tuzov, S., Havlin, S., and Bunde, A.: Recurrence intervals between earthquakes strongly depend on history, Physica A, 348, 591-595, 2005b.

Malamud, B. D. and Turcotte, D. L.: Self-affine time series: I. Generation and analyses, Adv. Geophys., 40, 1-90, 1999.

Matthews, M. V., Ellsworth, W. L., and Reasenberg, P. A.: A Brownian model for recurrent earthquakes, Bull. Seism. Soc. Am., 92, 2233-2250, 2002.

Newman, W. I. and Phoenix, S. L.: Time-dependent fiber bundles with local load sharing, Phys. Rev. E, 63, 021507, doi:10.1103/PhysRevE.63.021507, 2001.

Pelletier, J. D. and Turcotte, D. L.: Self-affine time series: II. Applications and models, Adv. Geophys., 40, 91-166, 1999.

Pennetta, C.: Distribution of return intervals of extreme events, Eur. Phys. J. B, 50, 95-98, 2006.

Rikitake, T.: Recurrence of great earthquakes at subduction zones, Tectonophys., 35, 335-362, 1976.

Rikitake, T.: Earthquake Forecasting and Warning, D. Reidel Publishing Co., Dordrecht, 402pp., 1982.

Rikitake, T.: Assessment of earthquake hazard in the Tokyo area, Japan, Tectonophys., 199, 121-131, 1991.

Rundle, J. B.: A Physical Model for Earthquakes, 2. Application to Southern-California, J. Geophys. Res., 93, 6255-6274, 1988.

Rundle, J. B., Rundle, P. B., Donnellan, A., and Fox, G.: GutenbergRichter statistics in topologically realistic system-level earthquake stress-evolution simulations, Earth Planets Space, 56, 761-771, 2004

Sanchez, R., Newman, D. E., and Carreras, B. A.: Waiting-time statistics of self-organized-criticality systems, Phys. Rev. Lett., 88, 068302, doi:10.1103/PhysRevLett.88.068302, 2002.

Savage, J. C.: Empirical earthquake probabilities from observed recurrence intervals, Bull. Seism. Soc. Am., 84, 219-221, 1994.
Shcherbakov, R., Yakovlev, G., Turcotte, D. L., and Rundle, J. B.: Model for the distribution of aftershock interoccurrence times, Phys. Rev. Lett., 95, 218501, doi:10.1103/PhysRevLett.95.218501, 2005.

Sieh, K., Stuiver, M., and Brillinger, D.: A more precise chronology of earthquakes produced by the San-Andreas fault in southern California, J. Geophys. Res., 94, 603-623, 1989.

Sornette, D. and Knopoff, L.: The paradox of the expected time until the next earthquake, Bull. Seism. Soc. Am., 87, 789-798, 1997.

Stauffer, D. and Aharony, A.: Introduction To Percolation Theory, 2nd ed., Taylor \& Francis, 181pp., 1992.

Turcotte, D., Malamud, B. D., Morein, G., and Newman, W. I.: An inverse-cascade model for self-organized critical behavior, Physica A, 268, 629-643, 1999.

Turcotte, D. L.: Self-organized criticality, Rep. Prog. Phys., 62, 1377-1429, 1999.

Turcotte, D. L. and Malamud, B. D.: Landslides, forest fires, and earthquakes: examples of self-organized critical behavior, Physica A, 340, 580-589, 2004.

Utsu, T.: Estimation of parameters for recurrence models of earthquakes, Bull. Earthquake Res. Insti.-Univ. Tokyo, 59, 53-66 1984.

Ward, S. N.: A synthetic seismicity model for southern California: Cycles, probabilities, and hazard, J. Geophys. Res., 101, 22393 22 418, 1996, 1996.

Ward, S. N.: San Francisco Bay Area earthquake simulations: A step toward a standard physical earthquake model, Bull. Seism. Soc. Am., 90, 370-386, 2000.

Working Group on California Earthquake Probabilities: Earthquake probabilities in the San Francisco Bay Region, 2002-2031, U.S. Geological Survey, Open-File Report 2003-214, 2003.

Yakovlev, G., Newman, W. I., Turcotte, D. L., and Gabrielov, A.: An inverse cascade model for self-organized complexity and natural hazards, Geophys. J. Int., 163, 433-442, 2005.

Yakovlev, G., Turcotte, D. L., Rundle, J. B., and Rundle, P. B.: Simulation based distributions of earthquake recurrence times on the San Andreas fault system, Bull. Seism. Soc. Am., 96, 19952007, 2006. 